\title{
Calculation of Solar Motion for Localities in the USA
}

\author{
Keith John Treschman \\ Science/Astronomy, University of Southern Queensland, Toowoomba, Australia
}

\author{
Email address: \\ treschmankm@bigpond.com
}

\section{To cite this article:}

Keith John Treschman. Calculation of Solar Motion for Localities in the USA. American Journal of Astronomy and Astrophysics. Vol. 9, No. 1, 2021, pp. 1-7. doi: 10.11648/j.ajaa.20210901.11

Received: December 17, 2020; Accepted: December 31, 2020; Published: January 12, 2021

\begin{abstract}
Even though the longest day occurs on the June solstice everywhere in the Northern Hemisphere, this is NOT the day of earliest sunrise and latest sunset. Similarly, the shortest day at the December solstice in not the day of latest sunrise and earliest sunset. An analysis combines the vertical change of the position of the Sun due to the tilt of Earth's axis with the horizontal change which depends on the two factors of an elliptical orbit and the axial tilt. The result is an analemma which shows the position of the noon Sun in the sky. This position is changed into a time at the meridian before or after noon, and this is referred to as the equation of time. Next, a way of determining the time between a rising Sun and its passage across the meridian (equivalent to the meridian to the setting Sun) is shown for a particular latitude. This is then applied to calculate how many days before or after the solstices does the earliest and latest sunrise as well as the latest and earliest sunset occur. These figures are derived for 60 cities in the USA. The selection was initially based on the most populous urban areas but was extended to ensure that each of the 50 states has a representative city.
\end{abstract}

Keywords: Solstice, Elevation, Obliquity, Elliptical Orbit, Meridian, Analemma, Equation of Time

\section{Introduction}

The June solstice is the longest day of the year in the USA and the December solstice is the shortest. However, calculations in this paper show that:

The earliest sunrise happens before the June solstice and the latest sunset after;

The latest sunrise happens after the December solstice and the earliest sunset before;

The effect at the December solstice is more pronounced than for the June one and

The number of days departure for earliest/latest sunrise/sunset from the longest and shortest days is latitudinally dependent and increases towards the equator.

\section{Dates Used in this Paper}

The solstices, equinoxes, perihelion (closest distance to Sun) and aphelion (furthest point from Sun) occur at instances and these are given in Universal Time, the time at Greenwich, England. The USA has a wide spread of time zones from 5-10 hours behind Greenwich. A solstice, for example, happens at an instant and may occur on different days in different time zones. Calculations in this paper are based on Central Standard Time of -6 hours difference, with Chicago being the representative locality. 2022 is the year selected. One advantage to this is that it avoids a leap year. So, relevant orbital and seasonal dates chronologically for the USA in 2022 Astropixels [1] are shown in Table 1.

Table 1. Dates in 2022 for USA Central Standard Time for solar occurrences.

\begin{tabular}{ll}
\hline Solar Occurrence & Date in 2022 \\
perihelion & January 04 \\
equinox & March 20 \\
solstice & June 21 \\
aphelion & July 04 \\
equinox & September 22 \\
solstice & December 21 \\
\hline
\end{tabular}

\section{Outline of Paper}

1. The elevation of the Sun at noon over a year is related to the tilt of the spin axis of Earth which is inclined at $23^{\circ} .44$ Seidelman_2006 [2] to the vertical of its orbital plane (obliquity). This is determined graphically.

2. The east-west variation of the Sun from the meridian at noon depends on two factors. These are an elliptical rather than a circular orbit for Earth and the obliquity. These factors 
are independent of each other. This derivation is analyzed in steps (as in Yeow_2002 [3]) with first taking only the elliptical orbit and setting the obliquity as $0^{\circ}$.

3. Second, the influence of the obliquity under a circular orbit is found.

4. Third, the two factors from 3.2 and 3.3 are combined.

5. The result from 3.4 is joined with that from 3.1 to display the angle of the Sun and its east-west variation from the meridian over a year.

6. 3.5 is converted from an angle to time in minutes to produce the Equation of Time.

7. Rising and setting times may be calculated for one's latitude.

\subsection{Variation of the Position of the Sun over the Year: Elevation}

The basic unit for the calendar is the time between successive March equinoxes and its duration is 365.24219 days [2]. From the point of view of Earth, the average angular movement of the Sun is

$$
360^{\circ} / 365.24219 \text { days }=0^{\circ} .986 \text { per day. }
$$

Declination is the angle from the celestial equator moving away from it at right angles with + to the north and - to the south. Over the year

$$
\text { declination of Sun=23.44 } \sin (0.986(286+D))
$$

where 286 is the number of days from the March equinox of the previous year (here March 202021 [1]) to 31 December, and $\mathrm{D}$ is the number of days from 01 January in the current year (2022).

In Excel, highlight column A, Format Cells to Custom and d-mmm. In cell A1 place 1/01/2022 and in A2 5/01/2022. These will change to 1-Jan and 5-Jan respectively. In A3 place $=A 2+5$ and copy to $31-D e c$. In $B 1$ and $B 2$ respectively place 1 and 5. In $B 3$ put $=B 2+5$ and copy to 365 . In $C 1,=23.44 * \operatorname{SIN}(\operatorname{RADIANS}(0.986 *(286+B 1)))$. Copy will be taken for granted from now. Graph $C$ against $B$ to give Figure 1 .

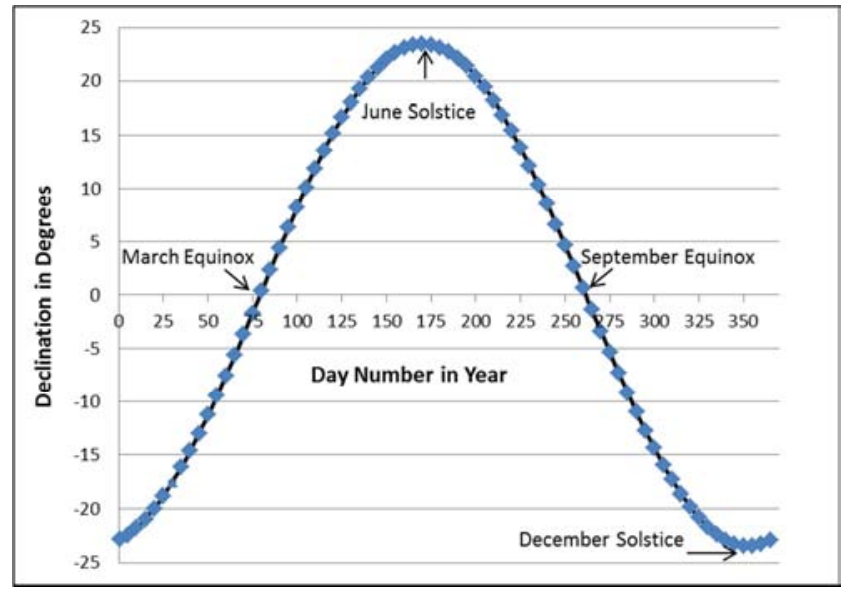

Figure 1. Declination of the Sun over a Year.
Figure 2 shows the declination taken from Earth's equator and an observer in the northern hemisphere.

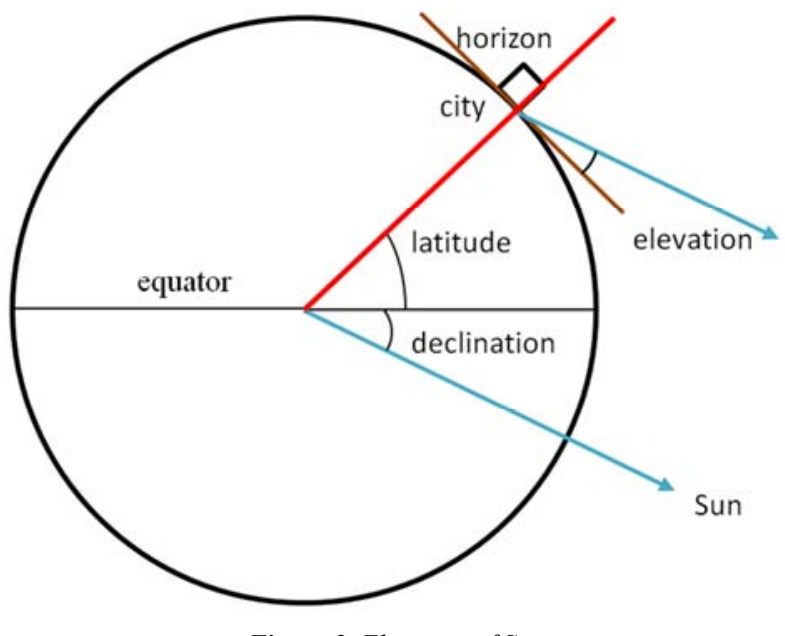

Figure 2. Elevation of Sun.

The upper blue line is parallel to the lower one. On this scale, the Sun is far enough away that the upper parallel line also points to the Sun. By convention, declinations south of the equator are negative. Hence, from geometry for the Sun in either hemisphere

$$
\text { elevation }=90^{\circ}-(\text { latitude }- \text { declination }) .
$$

For a fixed latitude, the elevation of the Sun at noon varies due to the declination.

In D1,=90-(latitude-C1) where one's latitude in decimal degrees is used. Graph $D$ against $B$ for Figure 3. The example here uses Chicago $41.84\left(41^{\circ} .84 \mathrm{~N}\right)$.

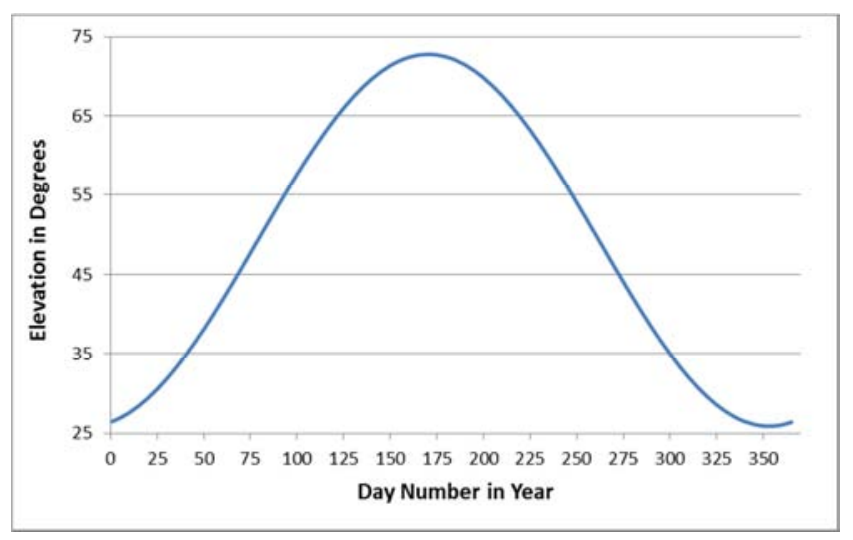

Figure 3. Elevation of Sun at Noon for Chicago over a Year.

\subsection{Deviation from Meridian for Elliptical Orbit Only}

The derivation following [3] fixes the tilt angle at $0^{\circ}$. If $N$ is the number of days after perihelion, $m=0.986 N$ (1) gives the angular change from perihelion of a uniformly moving Sun on a circle centered on Earth. The position of the real Sun uses Earth at one focus of an ellipse. The following equation gives the angular change $v$ of the actual Sun in an elliptical orbit Duffet-Smith 1992 [5] where the eccentricity $e$ of Earth's orbit is 0.01671 [2]. 


$$
\nu=m+(360 / \pi) e \sin m=0.986 \mathrm{~N}+1.915 \sin (0.986 \mathrm{~N})
$$

In $E 1,=B 1-4 \quad$ (Jan 04 is perihelion). In $F 1,=0.986^{*} E 1+1.915 * \operatorname{SIN}\left(\operatorname{RADIANS}\left(0.986^{*} E 1\right)\right) . \quad$ In $G 3,=(F 3-F 2) / 5$. This is the average each 5 days. Plot $G$ versus $B$ from cell 3 for Figure 4. Add dotted horizontal line at 0.986 .

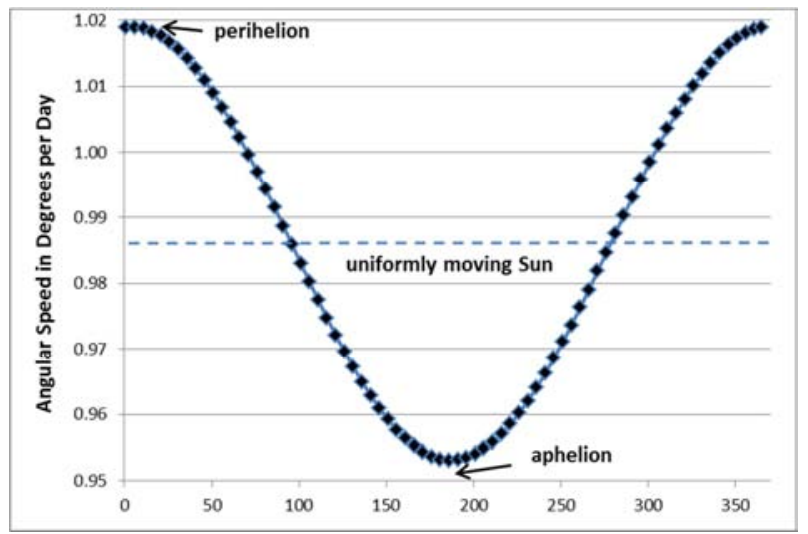

Figure 4. Angular Speed of Sun in an elliptical orbit compared with a uniformly moving Sun.

The elliptical orbit is based on the first law of planetary motion of Kepler. His second law is demonstrated in Figure 4 by the maximum speed at perihelion and the minimum at aphelion.

Equation (4) may be rearranged so that

$$
Z_{I}=m-v=-1.915 \sin (0.986 \mathrm{~N})
$$

is the angular difference between a circular and elliptical orbit for the Sun. A positive value gives a faster moving Sun so that at noon it is west of the meridian. Conversely, a negative value gives a slower moving Sun, which at noon is east of the meridian. Thus, $Z_{l}$ represents the position of the Sun relative to the meridian based on the elliptical orbit.

In $H 1,=-1.915 * \operatorname{SIN}(R A D I A N S(0.986 * E 1))$. Graph $H$ against $B$ for Figure 5 .

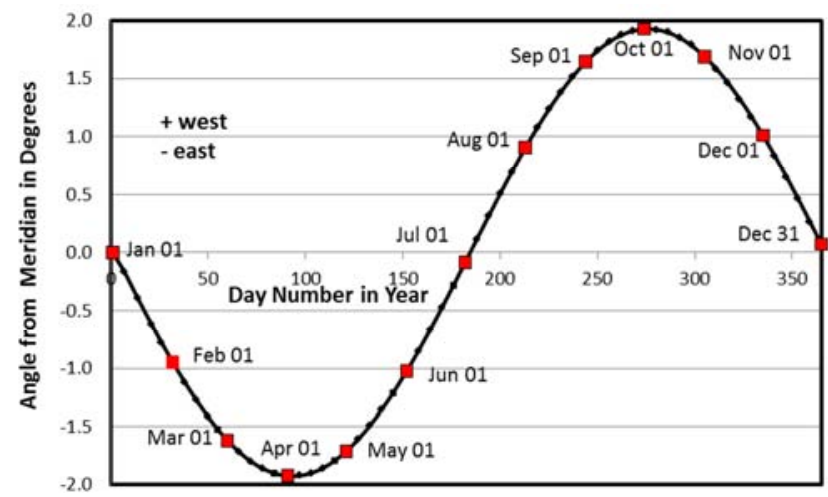

Figure 5. Angle from meridian for factor of elliptical orbit only.

\subsection{Deviation from Meridian for Obliquity Only}

A circular orbit is selected. The celestial equator and ecliptic are inclined at $23^{\circ} .44$ to each other in Figure 6.

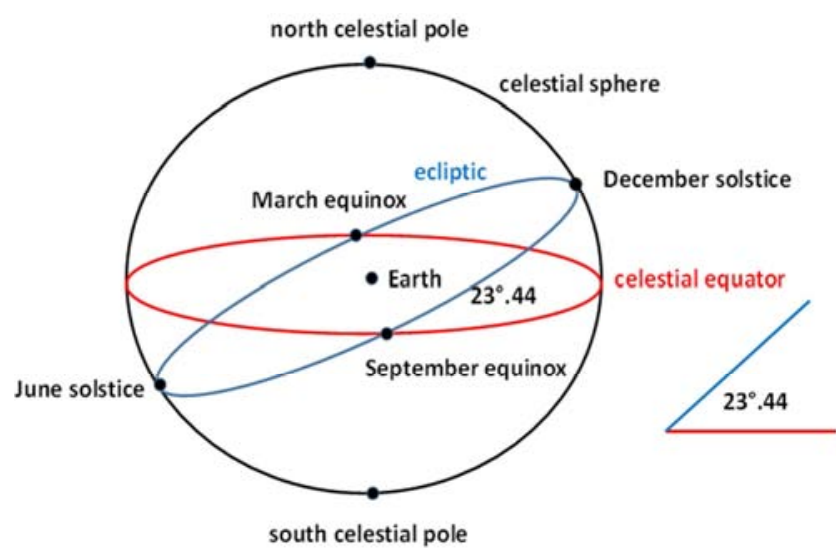

Figure 6. Celestial Sphere with Celestial Equator and Ecliptic.

Movement on the celestial equator is the basis for clock time, but the real Sun moves at an angle to this. From Figure $6, \cos 23^{\circ} .44=0.917$ so that for a $1^{\circ}$ movement of the Sun along the ecliptic, its component on the celestial equator is $0^{\circ} .917$. The "real Sun" and the "clock Sun" meet at the equinoxes. The real Sun and the clock Sun are moving parallel at the solstices, so they are again in step. The maximum difference will be halfway between them, that is, at $45^{\circ}$ along the orbit. The difference in angular movement at $45^{\circ}$ gives the amplitude of a graph due to this factor only. Spherical geometry Kaler [6] is necessary to show the

angular movement on the ecliptic $=\arctan (0.917$ tan $\left.45^{\circ}\right)=42^{\circ} .52$ Mills_978 [7]

$$
45^{\circ}-42^{\circ} .52=2^{\circ} .48 \text {. }
$$

While the period for the elliptical factor is one year, it is 6 months (182.5 days) for the obliquity factor. In 2022 there are 75 days between perihelion and the March equinox. One quarter of a year is 91.25 days. If $N$ is taken as previously, the effect here is [3]

$$
\mathrm{Z}_{2}=2.48 \sin \left(\frac{(\mathrm{N}-75) 182.5}{91.25}\right) .
$$

In $I 1,=2.48 * \operatorname{SIN}(R A D I A N S((E 1-75) * 182.5 / 91.25))$. Plot I against $B$ in Figure 7.

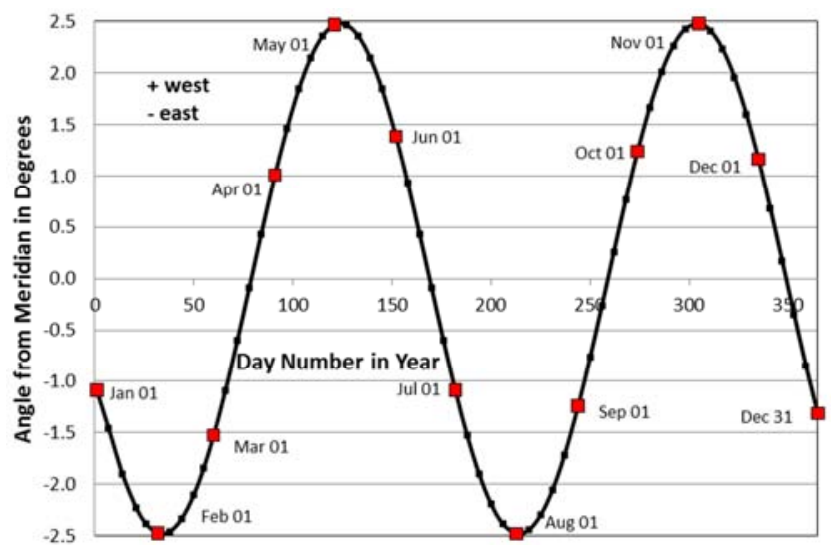

Figure 7. Angle from meridian for obliquity only. 


\subsection{Deviation from Meridian for Elliptical Orbit and Obliquity Combined}

The two effects of elliptical orbit and tilt angle are now combined from (5) and (8).

$$
\begin{gathered}
Z=\mathrm{Z}_{1}+\mathrm{Z}_{2} \\
Z=-1.915 \sin 0.986 \mathrm{~N}+2.48 \sin \left(\frac{(\mathrm{N}-75) 182.5}{91.25}\right) .
\end{gathered}
$$

In $J 1,=H 1+I 1$. Graph $J$ (combined angle from meridian) against B in Figure 8.

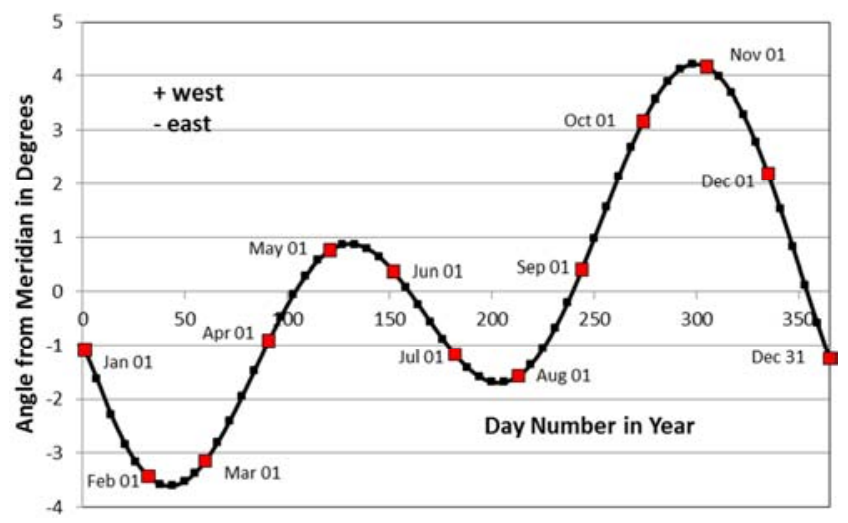

Figure 8. Angle from meridian for elliptical orbit and obliquity.

\subsection{Elevation and Deviation from the Meridian Combined}

The union of the elevation for a specific locality (Figure 3) and deviation from the meridian (Figure 8) for the Sun results in the construction of an analemma, which is in the shape of a figure eight. The word is derived from a pedestal for a sundial. This is what would be observed for the noon Sun over a year at a specific place. However, a general analemma is constructed by having the vertical axis the declination (Figure 1) and the horizontal axis as the angle of the noon Sun from the meridian (Figure 8 ) with positive values west and negative ones east.

Plot $C$ against $J$ for Figure 9.

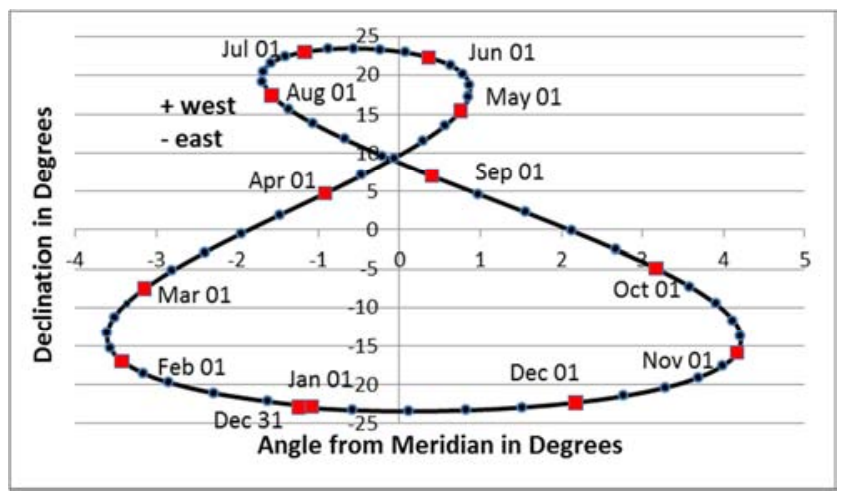

Figure 9. Analemma.

The actual position in the sky is obtained by raising or lowering the entire curve to match the elevation at one's latitude.

As the Sun is not on the meridian at noon at the solstices, the analemma is skewed and does not line up with the vertical axis.

\subsection{Equation of Time}

The sidereal rotation of the Earth is 23 hours 56 minutes 04 seconds $=0.997269$ days.

0.997269 days $\times 24 \times 60 / 360^{\circ}=3.989$ minutes per degree. $(11$

Thus, from the position of the Sun given as an angle, one may determine the time earlier or later than noon that it crosses the meridian (Figure 8 into Figure 10). For the Sun west of the meridian at noon, it is faster than a clock and will be shown here as a positive time.

In K1,=3.989*J1. Graph K against B for Figure 10.

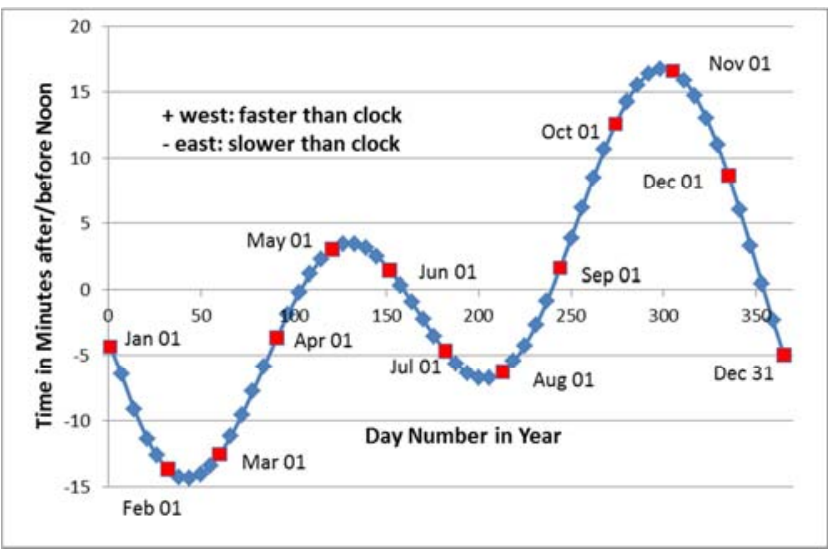

Figure 10. Equation of Time.

The relative sizes of the crests show the effect in December is more pronounced than in June.

\subsection{Rising and Setting Times for One's Latitude}

The time from sunrise to its crossing of the meridian (and from the meridian to sunset) depends on both the declination of the Sun and the latitude of the observer. Even though the longest and shortest days are generally the same date for all places in the USA, earliest and latest sunrise and sunset are latitudinally dependent. Houston Texas, being close to the equator, is used as an example as this gives a large difference in days. However, adopt your own latitude. Then, the number of days before or after the solstices for earliest/latest sunrise and sunset is calculated for your locality.

The algorithm for the time interval between the rising of a star (here the Sun) and its transit over the observer's meridian (or equivalently from the meridian to setting) for particular declinations is derived firstly by determining an angle from spherical geometry [6] and is Ridpath [8]

$\cos ($ semi-diurnal arc $)=-\tan ($ declination $) \times \tan$ (latitude) $(12)$ where the semi-diurnal arc is the angle that a star makes from either horizon to the meridian. Then, a general formula for converting this angle to length of time is

time in minutes=semi-diurnal arc x 3.989 (equation 11). (13) 
In L1,=DEGREES(ACOS(-TAN(RADIANS(C1))

*TAN(RADIANS(latitude))))*3.989

where Houston, Texas of latitude $29.79\left(29^{\circ} .79 \mathrm{~N}\right)$ is used. Graph L against B for Figure 11.

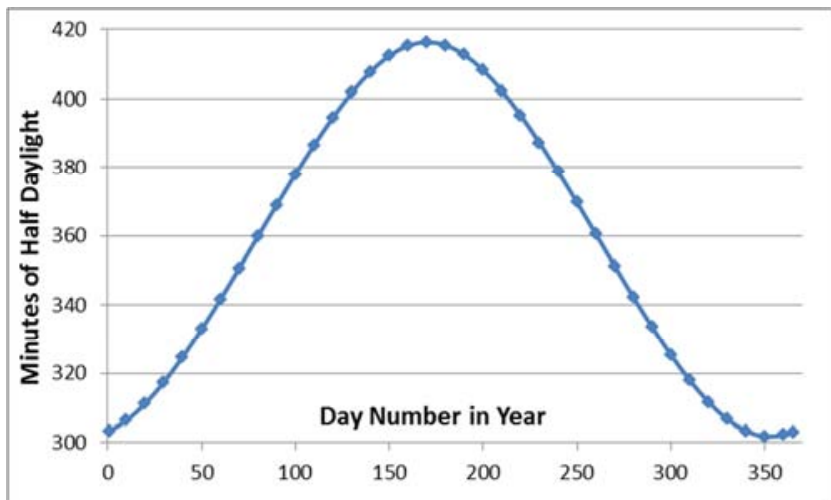

Figure 11. Time between horizon and meridian transit for Houston Texas over year.

The following procedure Wagon_1990 [9] is based on the semi-diurnal time for Houston, Texas, but use your own latitude.

Addition of the minutes before or after noon in Figure 10 to the minutes of half daylight in Figure 11 gives the actual minutes before noon of sunrise.

In $M 1,=K 1+L 1$ for time of sunrise in minutes before noon. Graph M against B for Figure 12.

Subtraction of the minutes before or after noon in Figure 10 from the minutes of half daylight in Figure 11 gives the actual minutes after noon of sunset.

In N1, =L1-K1 for time of sunset in minutes after noon. Graph $N$ against $B$ for Figure 13 where the vertical axis is reversed to have a sense that the spacing between the plots in Figures 12 and 13 is the length of daylight.

Inspection of Figures 12 and 13 shows that the earliest sunrise precedes the June solstice and the latest sunset trails it, and the latest sunrise trails the December solstice, and the earliest sunset precedes it.

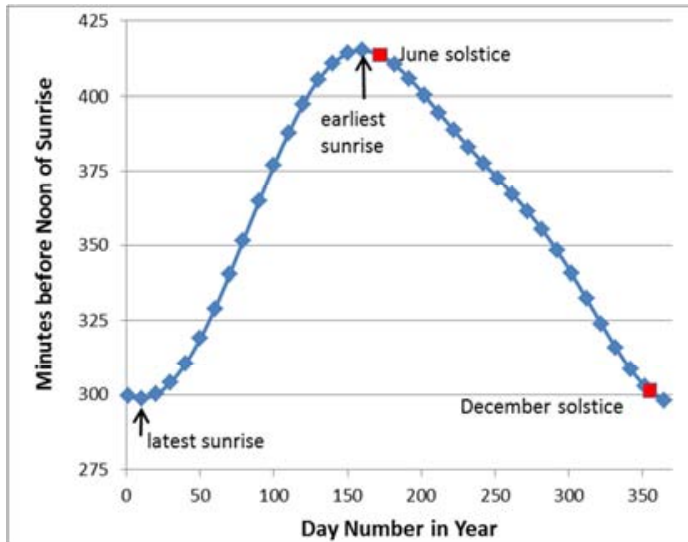

Figure 12. Time of sunrise in minutes before noon for Houston, Texas latitude $29^{\circ} .79 \mathrm{~N}$ for 2022 .

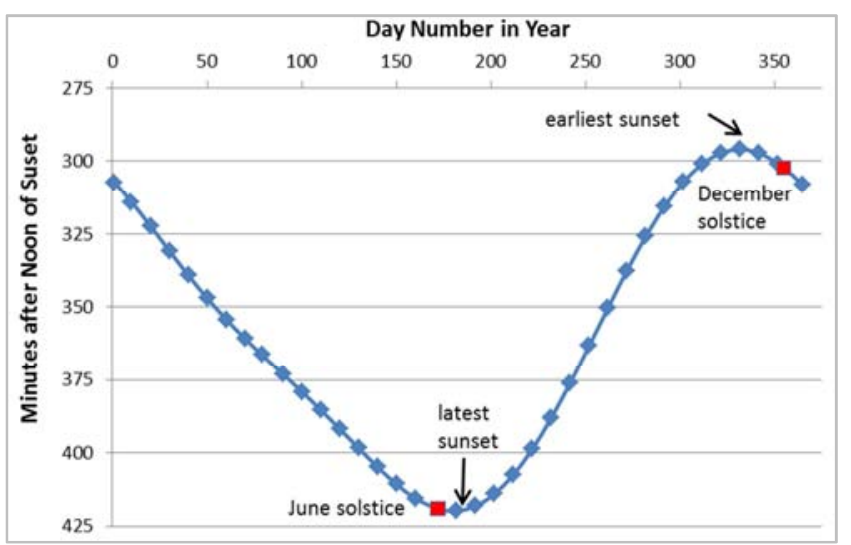

Figure 13. Time of sunset in minutes after noon with vertical axis reversed for Houston, Texas latitude $29^{\circ} .79 \mathrm{~N}$ for 2022.

Addition of the minutes before noon of sunrise (Figure 12) and minutes after noon of sunset (Figure 13) gives the length of daylight for each day.

$$
\text { In } \mathrm{O} 1,=\mathrm{M} 1+\mathrm{N} 1 .
$$

The data in Excel have been calculated for each 5 days. To ascertain the number of days difference from the solstices for the shortest/longest sunrise/sunset, the information can be set daily, but it is only necessary for June to early July 2022 and late November 2022 to early January 2023. As the effect is less pronounced further from the equator, these spans of the calendar could be even smaller. There is a slower rate of change at these maxima and minima, and it may be necessary to go to 3 decimal places to isolate one particular day. Also, these equations have some rounding effect for simplicity, and may differ ever so slightly from results put out from say, the United States Naval Office. Also, the clock time is for the longitude of a certain time zone. A difference in one's locality from this longitude introduces a minor adjustment.

From $O$ in these daily calculations, one should see that the figures do correspond to the longest and shortest days, given the caveats above. From $O$ also, one should determine the number of days of earliest sunrise before the June solstice, latest sunset after the June solstice, latest sunrise after the December solstice and earliest sunset before the December solstice.

The offset effect should be more pronounced for December than for June.

\section{Extra for the Mathematically Inclined}

The graphs in Figures 12 and 13 (for the daily calculations) could be lined one above the other. Let one be a function of $p$ against time $t$ and the other $q$ versus $t$. Let the vertical difference $q-p=r$. At the maximum and minimum values of $\mathrm{r}$, its differential $\mathrm{d} r / \mathrm{d} t=0$. Hence, $\mathrm{d} q / \mathrm{d} t-\mathrm{d} p / \mathrm{d} t=0$, and this will occur when these differentials are equal. This corresponds to the days when the slopes of these two curves are equal. Draw these slopes on the daily section of the graphs to verify this for the June solstice and then for the December solstice. They are shown for Houston, Texas at the June solstice for sunrise in figure 14 and sunset in figure 15 . The days are aligned if 
figure 14 is placed above figure 15 .

Table 2. Number of days for earliest sunrise before the June solstice for latest sunset after the June solstice latest sunrise after the December solstice earliest sunset before the December solstice for 60 USA cities arranged in decreasing order of latitude in degrees in decimal form.

\begin{tabular}{|c|c|c|c|c|c|c|c|}
\hline & city & state/district & latitude & $\mathbf{A}$ & B & $\mathbf{C}$ & D \\
\hline 1 & Anchorage & Alaska & 61.17 & 4 & 1 & 3 & 7 \\
\hline 2 & Seattle & Washington & 47.62 & 7 & 3 & 9 & 12 \\
\hline 3 & Fargo & North Dakota & 46.87 & 7 & 4 & 9 & 14 \\
\hline 4 & Billings & Montana & 45.79 & 7 & 4 & 9 & 14 \\
\hline 5 & Portland & Oregon & 45.54 & 7 & 4 & 10 & 14 \\
\hline 6 & Minneapolis & Minnesota & 44.96 & 8 & 4 & 10 & 15 \\
\hline 7 & Burlington & Vermont & 44.48 & 8 & 4 & 10 & 15 \\
\hline 8 & Portland & Maine & 43.66 & 8 & 4 & 10 & 15 \\
\hline 9 & Boise & Idaho & 43.60 & 8 & 4 & 10 & 15 \\
\hline 10 & Sioux Falls & South Dakota & 43.54 & 8 & 4 & 10 & 15 \\
\hline 11 & Milwaukee & Wisconsin & 43.06 & 8 & 4 & 11 & 16 \\
\hline 12 & Manchester & New Hampshire & 42.98 & 8 & 4 & 11 & 16 \\
\hline 13 & Detroit & Michigan & 42.38 & 8 & 5 & 11 & 16 \\
\hline 14 & Boston & Massachusetts & 42.33 & 8 & 5 & 11 & 16 \\
\hline 15 & Chicago & Illinois & 41.84 & 8 & 5 & 11 & 16 \\
\hline 16 & Providence & Rhode Island & 41.82 & 8 & 5 & 11 & 16 \\
\hline 17 & Des Moines & Iowa & 41.57 & 8 & 5 & 11 & 16 \\
\hline 18 & Omaha & Nebraska & 41.26 & 9 & 5 & 12 & 16 \\
\hline 19 & Bridgeport & Connecticut & 41.19 & 9 & 5 & 12 & 17 \\
\hline 20 & Cheyenne & Wyoming & 41.14 & 9 & 5 & 12 & 17 \\
\hline 21 & Salt Lake City & Utah & 40.78 & 9 & 5 & 12 & 17 \\
\hline 22 & Newark & New Jersey & 40.72 & 9 & 5 & 12 & 17 \\
\hline 23 & New York City & New York & 40.66 & 9 & 5 & 12 & 17 \\
\hline 24 & Philadelphia & Pennsylvania & 40.01 & 9 & 5 & 12 & 17 \\
\hline 25 & Columbus & Ohio & 39.99 & 9 & 5 & 12 & 17 \\
\hline 26 & Indianapolis & Indiana & 39.78 & 9 & 5 & 12 & 17 \\
\hline 27 & Denver & Colorado & 39.76 & 9 & 5 & 12 & 17 \\
\hline 28 & Wilmington & Delaware & 39.74 & 9 & 5 & 12 & 17 \\
\hline 29 & Baltimore & Maryland & 39.30 & 9 & 5 & 12 & 18 \\
\hline 30 & Kansas City & Missouri & 39.13 & 9 & 5 & 13 & 18 \\
\hline 31 & Washington & District of Columbia & 38.90 & 9 & 5 & 13 & 18 \\
\hline 32 & Charleston & West Virginia & 38.35 & 9 & 6 & 13 & 18 \\
\hline 33 & Louisville & Kentucky & 38.17 & 9 & 6 & 13 & 18 \\
\hline 34 & El Paso & Texas & 37.85 & 10 & 6 & 13 & 18 \\
\hline 35 & San Francisco & California & 37.73 & 10 & 6 & 13 & 18 \\
\hline 36 & Wichita & Kansas & 37.69 & 10 & 6 & 13 & 18 \\
\hline 37 & San Jose & California & 37.30 & 10 & 6 & 13 & 19 \\
\hline 38 & Virginia Beach & Virginia & 36.78 & 10 & 6 & 14 & 19 \\
\hline 39 & Las Vegas & Nevada & 36.23 & 10 & 6 & 14 & 19 \\
\hline 40 & Nashville & Tennessee & 36.17 & 10 & 6 & 14 & 19 \\
\hline 41 & Oklahoma City & Oklahoma & 35.47 & 10 & 6 & 14 & 19 \\
\hline 42 & Charlotte & North Carolina & 35.21 & 10 & 6 & 15 & 20 \\
\hline 43 & Albuquerque & New Mexico & 35.11 & 10 & 6 & 15 & 20 \\
\hline 44 & Memphis & Tennessee & 35.10 & 10 & 7 & 15 & 20 \\
\hline 45 & Little Rock & Arkansas & 34.73 & 11 & 7 & 15 & 20 \\
\hline 46 & Los Angeles & California & 34.02 & 11 & 7 & 15 & 21 \\
\hline 47 & Columbia & South Carolina & 34.00 & 11 & 7 & 15 & 21 \\
\hline 48 & Atlanta & Georgia & 33.76 & 11 & 7 & 15 & 21 \\
\hline 49 & Phoenix & Arizona & 33.57 & 11 & 7 & 15 & 21 \\
\hline 50 & Birmingham & Alabama & 33.53 & 11 & 7 & 15 & 21 \\
\hline 51 & San Diego & California & 32.82 & 11 & 7 & 16 & 21 \\
\hline 52 & Dallas & Texas & 32.79 & 11 & 7 & 16 & 21 \\
\hline 53 & Fort Worth & Texas & 32.78 & 11 & 7 & 16 & 21 \\
\hline 54 & Jacksonville & Mississippi & 32.32 & 11 & 7 & 16 & 21 \\
\hline 55 & Jacksonville & Florida & 30.34 & 12 & 8 & 17 & 22 \\
\hline 56 & Austin & Texas & 30.30 & 12 & 8 & 17 & 23 \\
\hline 57 & New Orleans & Louisiana & 30.05 & 12 & 8 & 17 & 23 \\
\hline 58 & Houston & Texas & 29.79 & 12 & 8 & 18 & 23 \\
\hline 59 & San Antonio & Texas & 29.47 & 13 & 8 & 18 & 23 \\
\hline 60 & Honolulu & Hawaii & 21.32 & 17 & 12 & 23 & 30 \\
\hline
\end{tabular}




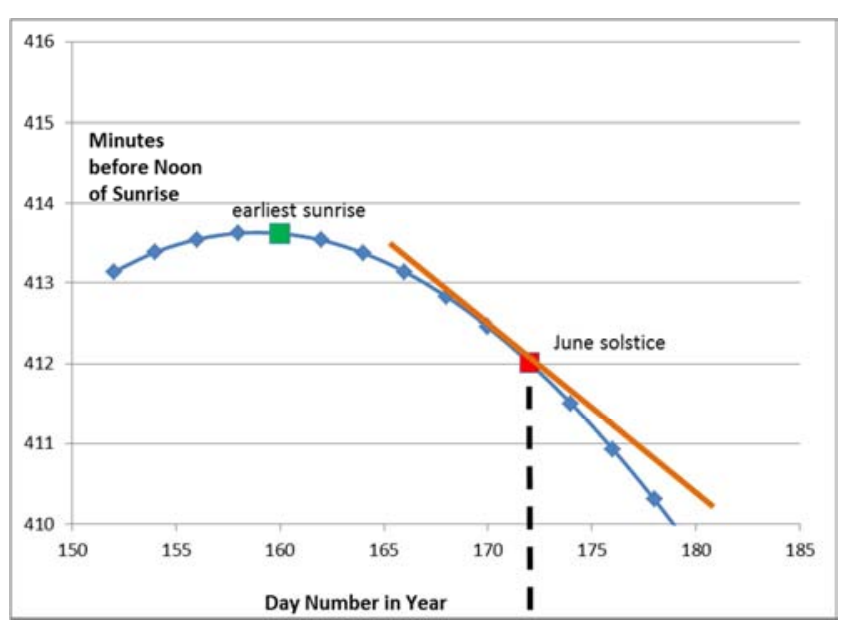

Figures 14. Times of sunrise for Houston, Texas latitude $29^{\circ} .79 \mathrm{~N}$ for 2022 around the June solstice to show the longest day and the equal slopes when placed above figure 15 .

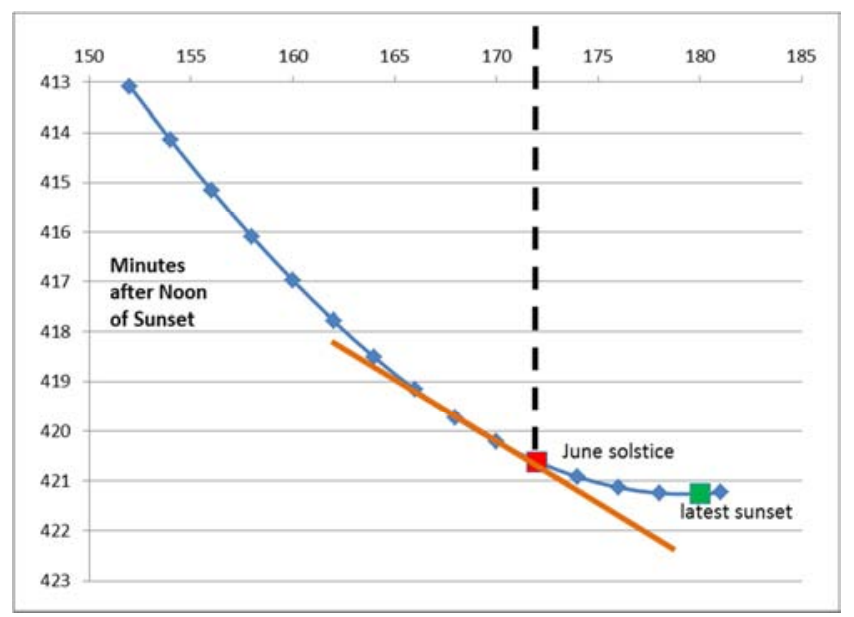

Figures 15. Times of sunset for Houston, Texas latitude $29^{\circ} .79$ N for 2022 around the June solstice to show the longest day and the equal slopes.

\section{Data for 60 Cities for Calculation of Solar Motion for Localities in the USA}

60 cities of the USA were selected for analysis. The 25 most populous urban areas from the estimated population at the end of 2016 were in the first group included. Then, for any state not represented in this collection, its most populous city was chosen. These data were actually calculated for 1999 , and while there is a slight variation from year to year, the pattern should be similar to the table.

\section{Conclusion}

The longest and shortest days are the same throughout the USA but these do NOT correspond to earliest/latest sunrise/sunset. This resulted in the following observations from calculations.

The earliest sunrise occurs before the June solstice and the latest sunset after.

The latest sunrise occurs after the December solstice and the earliest sunset before.

The effect at the December solstice is more pronounced than for the June one.

The effect increases with decreasing latitude.

\section{References}

[1] astropixels.com/ephemeris/astrocal/astrocal2020cst.html.

[2] P. Kenneth Seidelmann (ed), Explanatory Supplement to the Astronomical Almanac, University Science Books, Sausalito California, 2006.

[3] Teo Shin Yeow, The Analemma for Latitudinally-Challenged People, http://www.math.nus.edu.sg/aslaksen/projects/tsy.pdf, Singapore, 2002.

[4] D. Fletcher, Solar Declination, http://holodeck.st.usm.edu/vrcomputing/vic_t/tutorials/solar/d eclination.shtml, 2002.

[5] Peter Duffett-Smith, Practical Astronomy with your Calculator, $3^{\text {rd }}$ edition, Cambridge University Press, 1992.

[6] James B. Kaler, The Ever-Changing Sky - A Guide to the Celestial Sphere, Cambridge University Press, Cambridge, 2002.

[7] H. R. Mills, Positional Astronomy and Astro-Navigation Made Easy, Stanley Thornes Ltd., 1978.

[8] Ian Ridpath (ed), Norton's 2000.0 Star Atlas and Reference Handbook, $18^{\text {th }}$ edition, Longman Group UK Ltd. Essex.

[9] Stan Wagon, "Why December 21 is the Longest Day of the Year", Mathematics Magazine, 63, 1990, 307-311. 